\title{
221 K Local Photothermal Heating in a Si Plasmonic Waveguide Loaded with a Co Thin Film
}

\author{
Nana Ota ${ }^{1}$, Tomohiro Miyauchi ${ }^{2}$ and Hiromasa Shimizu ${ }^{1,3, *}$ \\ 1 Department of Electrical and Electronic Engineering, Tokyo University of Agriculture and Technology, \\ Koganei, Tokyo 184-8588, Japan; s218114t@st.go.tuat.ac.jp \\ 2 Department of Industrial Technology and Innovation, Tokyo University of Agriculture and Technology, \\ Koganei, Tokyo 184-8588, Japan; miyatuat@gmail.com \\ 3 Department of Applied Physics and Chemical Engineering, Tokyo University of Agriculture and Technology, \\ Koganei, Tokyo 184-8588, Japan \\ * Correspondence: h-shmz@cc.tuat.ac.jp; Tel.: +81-42-388-7996
}

Citation: Ota, N.; Miyauchi, T.;

Shimizu, H. 221 K Local

Photothermal Heating in a Si

Plasmonic Waveguide Loaded with a

Co Thin Film. Sensors 2021, 21, 6634.

https://doi.org/10.3390/s21196634

Academic Editor:

Zbigniew Jaroszewicz

Received: 26 August 2021

Accepted: 2 October 2021

Published: 6 October 2021

Publisher's Note: MDPI stays neutral with regard to jurisdictional claims in published maps and institutional affiliations.

Copyright: (c) 2021 by the authors. Licensee MDPI, Basel, Switzerland. This article is an open access article distributed under the terms and conditions of the Creative Commons Attribution (CC BY) license (https:// creativecommons.org/licenses/by/ $4.0 /)$.

\begin{abstract}
Photothermal heaters are important devices for optical switches and memories based on the thermo-optic/magneto-optic effect and phase change materials. We demonstrated photothermal heating in Si plasmonic waveguides loaded with Co thin films by measuring the resistance change upon inputting transverse-magnetic (TM) mode light. Temperature rise is proportional to the light intensity with clear polarization dependence. The photothermal conversion efficiency was estimated at $36 \mathrm{~K} / \mathrm{mW}$ and maximum temperature rise was estimated at $221 \mathrm{~K}$ at steady state upon the inputting $6.3 \mathrm{~mW}$ TM mode light for the $400 \mathrm{~nm}$-wide, $8 \mu \mathrm{m}$-long and $189 \mathrm{~nm}$-thick Co film deposited on the $\mathrm{Si}$ wire waveguide with $129 \mathrm{~nm}$-thick $\mathrm{SiO}_{2}$ buffer layer. The method to increase the efficiency is discussed based on the experimental and simulation results considering the thickness of the $\mathrm{SiO}_{2}$ buffer layer, Co layer and Si core layer, waveguide width, and wavelength. Local photothermal heaters in this study can be applied to a variety of fields including optical switches/memories without electrical control signals in photonic integrated circuits, on-chip optical sensors, and a lab-on-a-chip in biology, chemistry, and medicine.
\end{abstract}

Keywords: optical waveguide; plasmonic waveguide; local heating; photonic integrated circuit; optical sensors; surface plasmon polariton; photothermal effect

\section{Introduction}

Recent development of information and communication technology requires larger and larger bandwidth. Optical interconnects based on photonic integrated circuits (PICs) meet this demand and will play important roles in overcoming the limited bandwidth of electrical circuits. However, there are two problems for a dense integration of optical devices. One problem is the size of optical components. The length of an optical component is limited by the light wavelength, the relatively long length of interaction between light and matter (e.g., the electro-optical (EO), magneto-optical (MO), and thermo-optical (TO) interaction) and the large minimal bending radius of an optical waveguide. Another problem is realization of the optical devices without an electrical control signal in PICs for larger bandwidth. In particular, the realization of optical switches and memories, which can be operated by optical signals, is important in order to overcome the bandwidth of electrical circuits. Optical switches based on phase shifters with the TO effect have been reported with $100 \mu \mathrm{m}$-long Si waveguides and current application [1]. It is possible to realize heating in smaller area by combination of strong optical confinement and optical signals, without thermal crosstalk.

It is possible to reduce the size of optical components when an optical waveguide with a strong optical confinement such as a Si nanowire waveguide or a plasmonic waveguide are used [1-5]. The optical confinement in a Si nanowire waveguide is strong, because of 
a high refractive index contrast between $\mathrm{Si}$ and $\mathrm{SiO}_{2}$. Surface plasmon polaritons (SPPs) are quasiparticles by collective electron oscillations coupled to electromagnetic waves at the interface between materials having positive and negative permittivity, typically a dielectric and a metal. The use of a plasmonic waveguide benefits for a denser integration. Optical confinement in a plasmonic waveguide is stronger than in a Si nanowire waveguide, because the light is confined by a metal/dielectric interface beyond the diffraction limit. It makes the optical confinement stronger and enables stronger interaction between light and matter [5].

The interaction between light and matter with SPP has been applied to optical refractive index sensors with propagating/localized surface plasmon resonance (SPR) [6], localized surface plasmon with enhanced Raman scattering (SERS) [7], magnetic modulation of SPP and optical isolator application [8], and light-induced local heating with metal nanoparticles $[9,10]$. Light-induced local heating has been applied for thermophoretic manipulation of DNA with Au nanoparticles [11], resonant light-triggered DNA release from plasmonic nanoparticles [12], and selective light-induced contents release from liposomes [13]. It was reported that the liquids inside the micro- and nanochannels were heated to approximately $41^{\circ} \mathrm{C}$ using a laser power of $38 \mathrm{~mW}$ [11].

A Si waveguide combined with propagating SPP is one of the choices used in order to realize a lot of functions such as light-guiding, optical modulating [14], polarization handling, heating, and optical sensing [6], in nanoscale integration. Propagating SPPs combined with a Si waveguide have the advantages of the capability of integrating light sources, detectors, and input/output optical fibers on a single chip in a scheme of PICs.

There have been reports on local heating in Si hybrid nanoplasmonic devices [5,15], where the joule heating by the propagation loss owing to the interaction between light and metal is used. The local heaters are important devices for optical switches based on the TO and MO effect [16], optical memories loaded with phase change materials [17-19], and a lab-on-a-chip for biology, chemistry, and medicine [11-13]. Heating to $200{ }^{\circ} \mathrm{C}$ is required for optical memory applications with phase change materials such as $\mathrm{Ge}_{2} \mathrm{Sb}_{2} \mathrm{Te}_{5}$ (GST) [17-19]. The magneto-optical disk employed MO materials (GdFeCo, and $\mathrm{TbFeCo}$ ) as recording media, and when the temperature is high enough to reach the Curie temperature of the MO materials $\left(\sim 200^{\circ} \mathrm{C}\right)$, their magnetization direction can be reversed and stored after cooling [16]. Heat-assisted magnetic recording (HAMR) uses heat from a laser beam confined below the diffraction limit ( $<50 \mathrm{~nm}$-wide), in order to write the media of large perpendicular magnetic anisotropy at near $450{ }^{\circ} \mathrm{C}$ [20].

In a previous study of $\mathrm{Si}$ hybrid nanoplasmonic devices, a heating efficiency of $214 \mathrm{~K} / \mathrm{mW}$ and heating with $340 \mathrm{~K}$ for a power consumption of $1.6 \mathrm{~mW}$ was theoretically reported in a $10 \mu \mathrm{m}$-long $\mathrm{Au}$ thin film loaded on $100 \mathrm{~nm}$-wide Si nanowire waveguide [5]. A propagation loss of $0.35 \mathrm{~dB} / \mu \mathrm{m}$ and a heating efficiency of $49 \mathrm{~K} / \mathrm{mW}$ were experimentally estimated in a Si nanowire ring waveguide resonator partially loaded with a $20 \mathrm{~nm}$-thick and $5 \mu \mathrm{m}$-long Ti film, by measuring the shift of the resonance wavelength for different optical intensities [15]. The rise time upon the light input is faster than the typical time span of sweeping the wavelength for measuring the resonance characteristics, such that it is difficult to estimate the amount of the temperature rise and investigate the influence of the length, width, and thickness of the metal thin film on the heating characteristics. It is important to measure the temperature rise and rise time more precisely for various applications.

Measurement of the resistance of the metal thin film deposited on top of the $\mathrm{Si}$ waveguide is one of the simpler methods used to estimate the temperature rise and local photothermal heating. In this paper, we report the fabrication of Si plasmonic waveguides loaded with Co thin films, whose metal resistance changes are measured by a pair of electrode pads. Estimated temperature rise is proportional to the light intensity with clear polarization dependence, and the thermal conversion efficiency was estimated to $36 \mathrm{~K} / \mathrm{mW}$ for transverse magnetic (TM) mode light. Maximum temperature rise was estimated to 
$221 \mathrm{~K}$ upon the inputting $6.3 \mathrm{~mW}$ TM mode light at steady state. The influence of the width of the Co thin film deposited on the Si waveguides was investigated.

\section{Design of the Si Plasmonic Waveguide Heater}

We show the design of Si plasmonic waveguide heaters loaded with Co thin films. Transition metals are more appropriate than noble metals from the viewpoints of local heating by the propagation loss of SPP. Resistor material such as $\mathrm{Ti}, \mathrm{W}$ or Co has an upper limit of temperature rise and is appropriate from the viewpoint of a long-term reliability. One of the important factors determining the propagation loss is the imaginary part of the relative dielectric constant. We selected $\mathrm{Co}$ as having relative permittivity, $-38.0+50.7 \mathrm{i}$ at a wavelength of $1550 \mathrm{~nm}$, based on [21]. Co is less oxidized than Fe, and ferromagnetic metal whose Curie temperature is $1380 \mathrm{~K}$. The temperature rise at the interface of the Co layer and the Si wire waveguide can be measured based on the temperature coefficient of the resistivity of Co $\left(7.0 \times 10^{-3} 1 / \mathrm{K}\right)$ [22]. Figure 1 shows the schematic image of the Si plasmonic waveguide heater with a pair of electrode pads for measuring the resistance. The width $w$ of the Si wire waveguide and Co film on top of the waveguide were set to 400, and $1000 \mathrm{~nm}$ in order to investigate the influence of the width and optical confinement at the metal/dielectric interface on the heating efficiency. An S-bend waveguide with a bending radius of $50 \mu \mathrm{m}$ is used as the input and output waveguide in order to discriminate the output light from the stray light by coupling the light by a pair of lensed fibers. The height $h$ of the Si wire waveguide is $250 \mathrm{~nm}$. A $200 \mathrm{~nm}$-thick Co thin film is used as the metal with $100 \mathrm{~nm}$-thick $\mathrm{SiO}_{2}$ layer on the $\mathrm{Si}$ wire waveguide in order to realize the plasmonic waveguide having a propagation loss of $1.0 \mathrm{~dB} / \mu \mathrm{m}$.
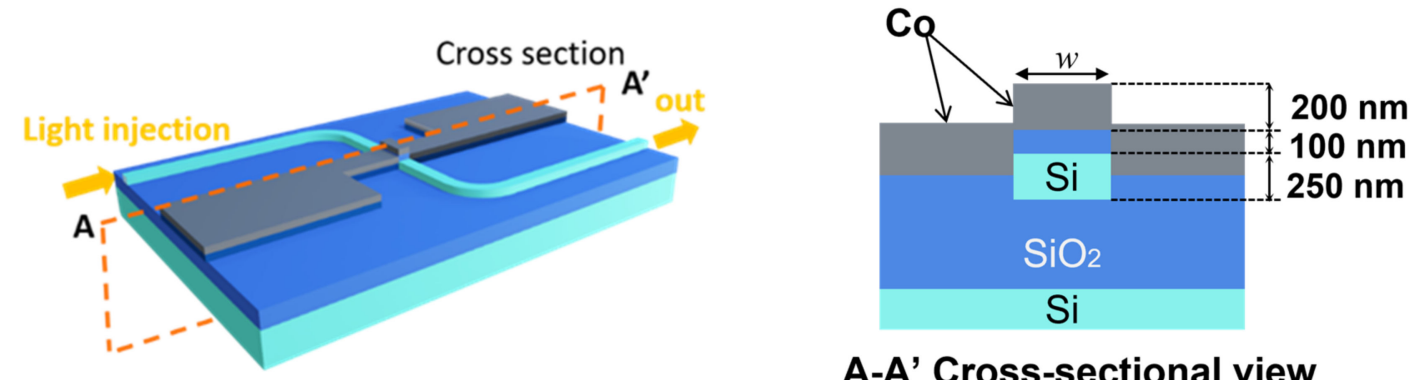

\section{A-A' Cross-sectional view}

(a)

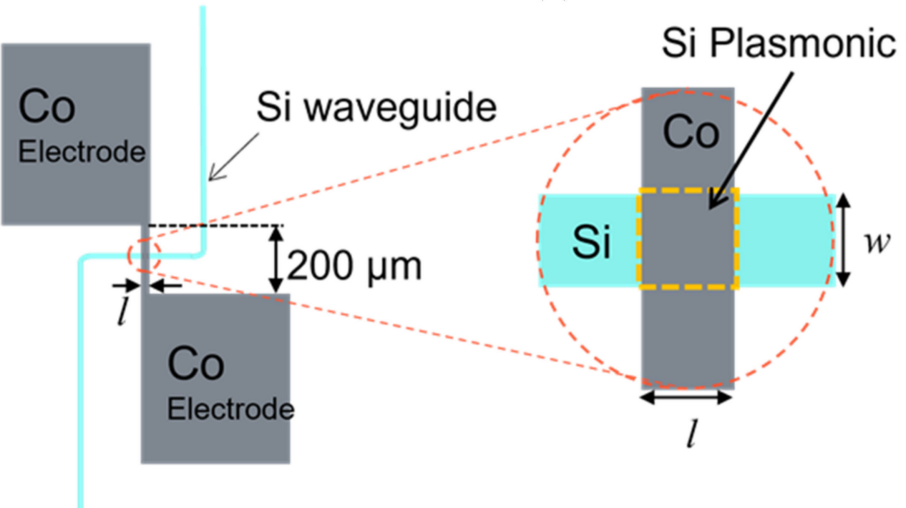

(b)

Figure 1. Schematic images of a Si plasmonic waveguide heater with the width of $w$, and length of $l$. (a) Entire image and cross-sectional image of the Si plasmonic waveguide with optical input waveguide and a pair of electrodes. (b) Dimension of the Si plasmonic waveguide heater loaded with a Co thin film, connected to a pair of electrode pads.

When all of the propagation loss is converted to Joule loss, the absorbed surface energy density is as large as $2.5 \times 10^{7} \mathrm{~W} / \mathrm{m}^{2}$ for input light intensity of $1 \mathrm{~mW}$ and $w=400 \mathrm{~nm}$, 
which is $2.5 \times 10^{4}$ times larger than that of the power density of sunlight at Air Mass 1.5. The length $l$ of the Co film on top of the Si wire waveguide was set at $8 \mu \mathrm{m}$.

\section{Fabrication of the Si Plasmonic Waveguide Heaters with Co Electrodes}

The designed waveguide heaters were fabricated on an SOI substrate by electronbeam (EB) writing and reactive ion etching. The $\mathrm{SiO}_{2}$ buffer layer and $\mathrm{Co}$ layer were deposited on the $\mathrm{Si}$ wire waveguides by sputtering and $\mathrm{EB}$ deposition with a lift-off process. Figure 2 shows the cross-sectional image taken by a scanning electron microscope (SEM). The thickness of the deposited $\mathrm{SiO}_{2}$ buffer layer was $129 \mathrm{~nm}$, which is slightly larger than the designed thickness $(100 \mathrm{~nm})$. The thickness of the Co thin film was $189 \mathrm{~nm}$, which is slightly smaller than the designed thickness $(200 \mathrm{~nm})$. Since the Co layer is also used as the electrical resistance, the Co film was formed at the both sides of the Si plasmonic waveguide and was connected with a pair of electrode pads $(0.40 \mathrm{~mm} \times 0.80 \mathrm{~mm})$ with a distance of $200 \mu \mathrm{m}$, as an optical microscope image shows in Figure 3. The distance between the both facets including the input and output waveguides is $3.5 \mathrm{~mm}$.

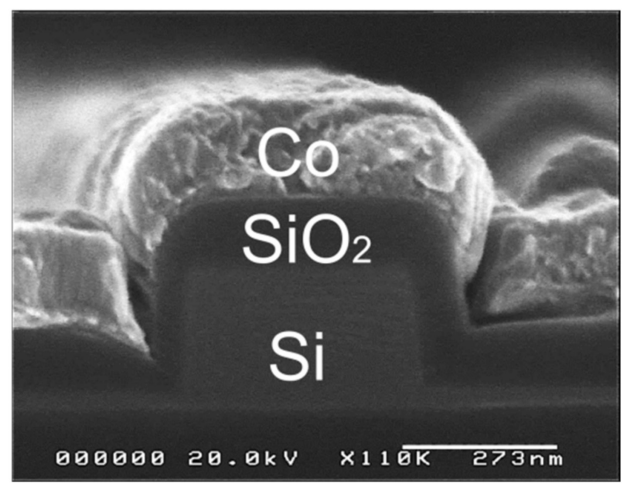

Figure 2. A cross sectional SEM image of Si plasmonic waveguide.

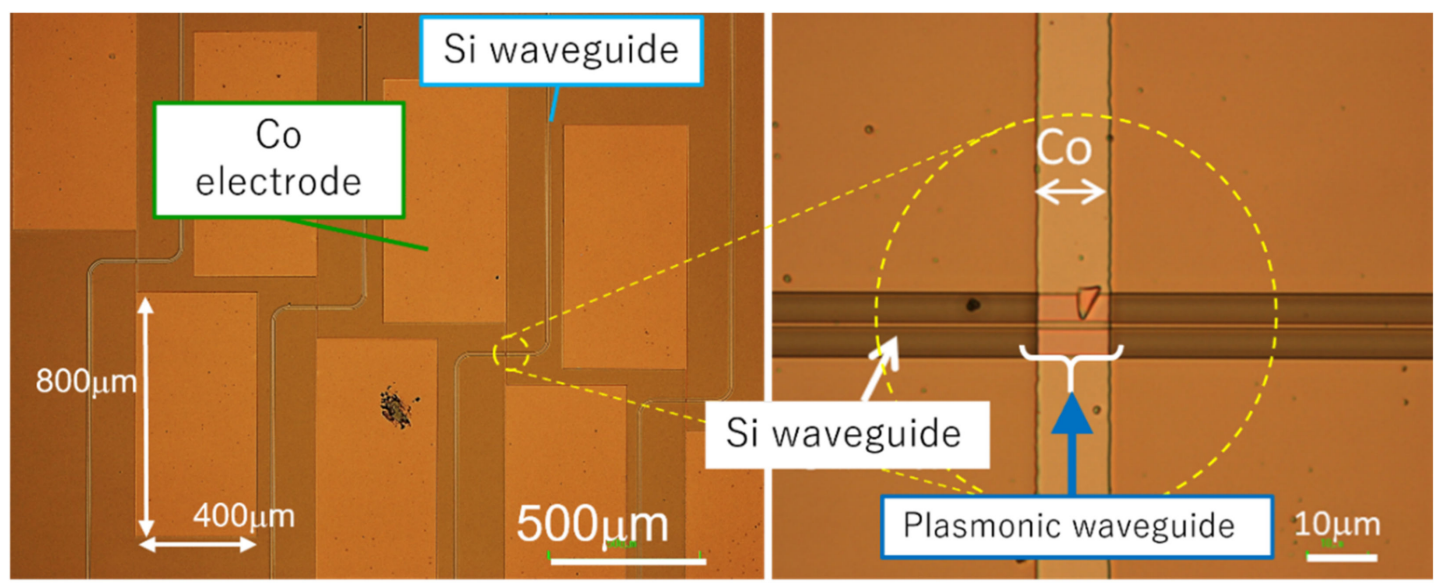

Figure 3. Optical microscope images of the Si plasmonic waveguide heater with electrode pads.

\section{Measurement of the Resistance Change and Rise Time for Characterization of the Photothermal Heating Efficiency}

First, we measured the temperature dependence of the resistance of the deposited Co thin film by two probes method with a digital multimeter as a reference of the local photothermal heating efficiency. Temperature of the whole waveguide was set between 15 and $45{ }^{\circ} \mathrm{C}$. The temperature of the whole waveguide was controlled by a thermoelectric controller. Figure 4 shows the temperature dependence of the resistance of the Co layer loaded on the $\mathrm{Si}$ waveguide $(w=400 \mathrm{~nm}$, and $l=8 \mu \mathrm{m})$ without light injection. The 
temperature coefficient of the resistance was $7.24 \times 10^{-2} \Omega / \mathrm{K}$, which is slightly larger than the estimated value $\left(5.2 \times 10^{-2} \Omega / \mathrm{K}\right)$ based on the temperature coefficient of the resistivity of Co [22], distance between the electrode pads $\left(l_{\mathrm{Co}}=200 \mu \mathrm{m}\right)$, width $(w=400 \mathrm{~nm})$, and thickness $(189 \mathrm{~nm})$ of the deposited Co thin film. One of the reasons for the deviation between the experimental and estimated values is that the surface of the deposited Co thin film is slightly oxidized and the resistivity is larger than the estimated value based on the ref. [21].

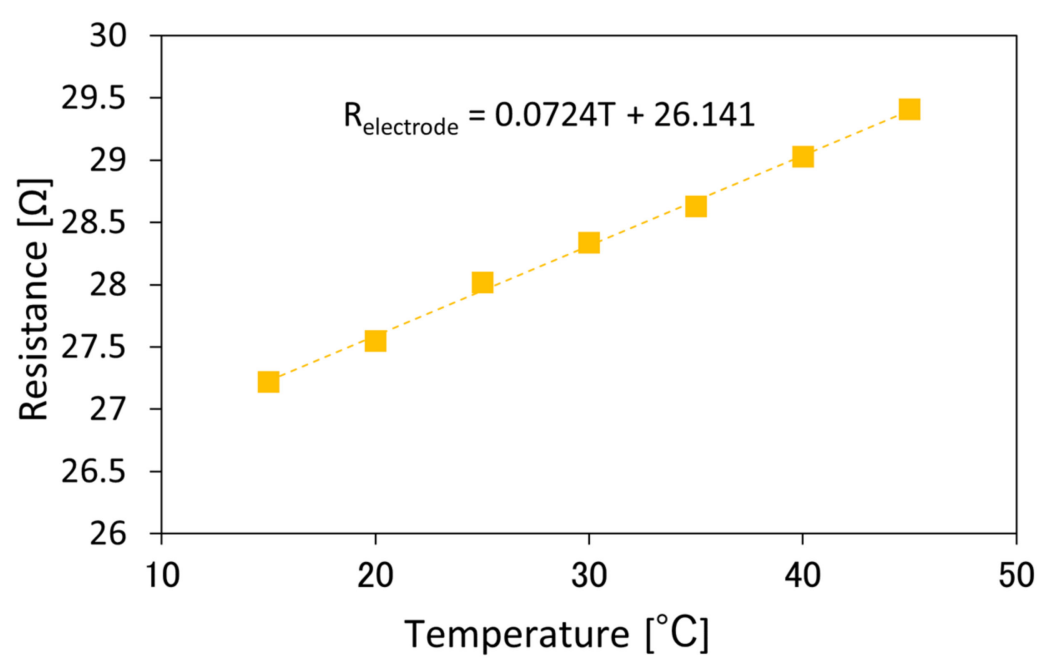

Figure 4. Temperature dependence of the resistance of the Co layer loaded on the Si waveguide $(w=400 \mathrm{~nm}$, and $l=8 \mu \mathrm{m})$ without light injection.

Then, we measured the resistance change of the Co layer upon the light injection and estimated the temperature rise. TM mode light with a wavelength of $1550 \mathrm{~nm}$ was coupled with a lens fiber for exciting SPP at the interface of $\mathrm{Co}$ and $\mathrm{SiO}_{2}$ layer. The temperature of the device before the measurement was $22.1^{\circ} \mathrm{C}$ (room temperature). No temperature control was applied to the device during the measurement. The resistance between the electrodes was measured with a digital multimeter, for intensity of 0 to $8 \mathrm{dBm}$ ( 1 to $6.3 \mathrm{~mW}$ ). Transverse electric (TE) mode light was also input for reference. The resistance before and after the light injection was recorded.

Figure 5 shows the resistance rise $\Delta R_{\text {light }}$ at steady state of the Co layer with the $\mathrm{Si}$ waveguides having the waveguide width $w$ of (a) 400, and (b) $1000 \mathrm{~nm}$, for TM and TE mode light. The resistance rise was larger with TM mode light than with TE mode light, and larger with narrower waveguide $(w=400 \mathrm{~nm})$ than wider waveguide $(w=1000 \mathrm{~nm})$. The resistance changes before and after the input of TM mode light is proportional to the input light intensity, and the efficiencies are $5.2 \times 10^{-3} \Omega / \mathrm{mW}$ for $w=400 \mathrm{~nm}$, and $1.9 \times 10^{-3} \Omega / \mathrm{mW}$ for $w=1000 \mathrm{~nm}$. The maximum resistance change $\Delta R_{\max }$ was $0.048 \Omega$ for $w=400 \mathrm{~nm}$, and $1.6 \mathrm{~s}$ after injection of $6.3 \mathrm{~mW}$ TM mode light, whereas the resistance change $\Delta R_{\text {steady }}$ was $0.032 \Omega$ for the same condition at steady state. When we assume that the resistance changed locally on the top of the waveguide (the area of $w \times l$ ) by light injection, and unchanged outside the waveguide, the local temperature rise $\Delta T_{\text {local }}$ for the Si plasmonic waveguide can be estimated from the temperature dependence of the resistance of the whole electrode $\left(\frac{\Delta R_{C_{0}}}{\Delta T}=7.24 \times 10^{-2} \Omega / K\right)$ of Figure 4 . Since the temperature dependence of the whole electrode is expressed as $\frac{\Delta R_{C_{0}}}{\Delta T}=\frac{\Delta \rho}{\Delta T} \frac{l_{C_{0}}}{l d}$, the local temperature dependence of the resistance of the Co thin film on top of the waveguide is expressed as $\frac{\Delta R_{\text {local }}}{\Delta T_{\text {local }}}=\frac{\Delta \rho}{\Delta T_{\text {local }}} \frac{w}{l d}$ and the temperature coefficient of the resistivity $\left(\frac{\Delta \rho}{\Delta T}, \frac{\Delta \rho}{\Delta T_{\text {local }}}\right)$ is constant, and can be derived by following Equation (1).

$$
\Delta T_{\text {local }}=\frac{1}{\frac{\Delta R_{\mathrm{Co}}}{\Delta T}} \times \Delta R_{\text {light }} \times \frac{l_{\mathrm{Co}}}{w}
$$




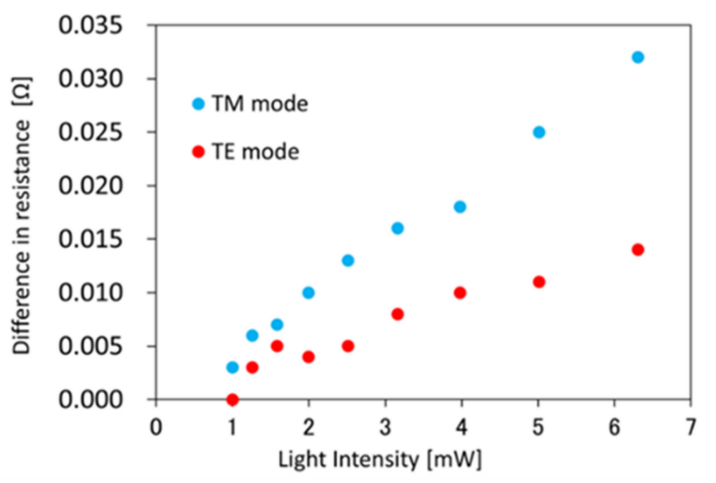

(a)

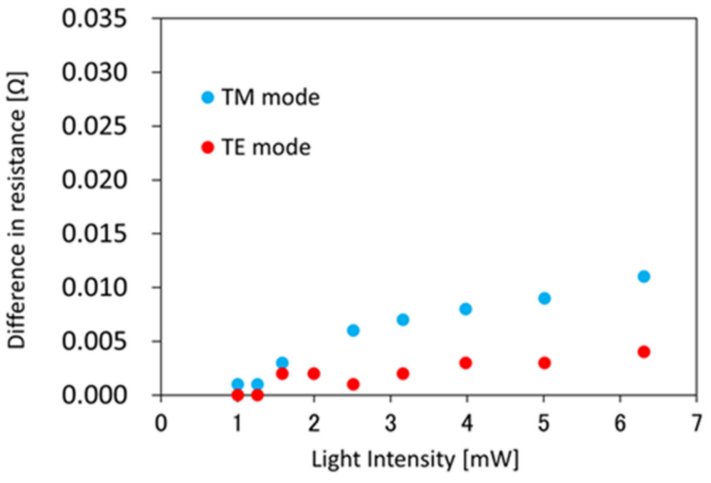

(b)

Figure 5. Difference in resistance $\Delta R_{\text {light }}$ of the Co layer with the Si waveguides having the waveguide width $w$ of (a) 400 , and (b) $1000 \mathrm{~nm}$, upon TM and TE mode light injection at steady state.

Figure 6 shows the estimated local temperature rise for TM and TE mode light with the $\mathrm{Si}$ waveguides having the waveguide width $w=$ (a) $400 \mathrm{~nm}$ and (b) $1000 \mathrm{~nm}$. Estimated temperature rise was $221 \mathrm{~K}$ for $6.3 \mathrm{~mW}$ TM mode light at steady state $\left(\Delta R_{\text {steady }}=0.032 \Omega\right)$. Please note that temperature of the device before the measurement was $22.1^{\circ} \mathrm{C}$, and the device is heated up to $22.1+221=243.1{ }^{\circ} \mathrm{C}$. The heating efficiency was estimated to $36 \mathrm{~K} / \mathrm{mW}$ and $16 \mathrm{~K} / \mathrm{mW}$ for TM and TE mode light. $\Delta R_{\max }=0.048 \Omega$ corresponds to temperature rise of $331 \mathrm{~K}$.

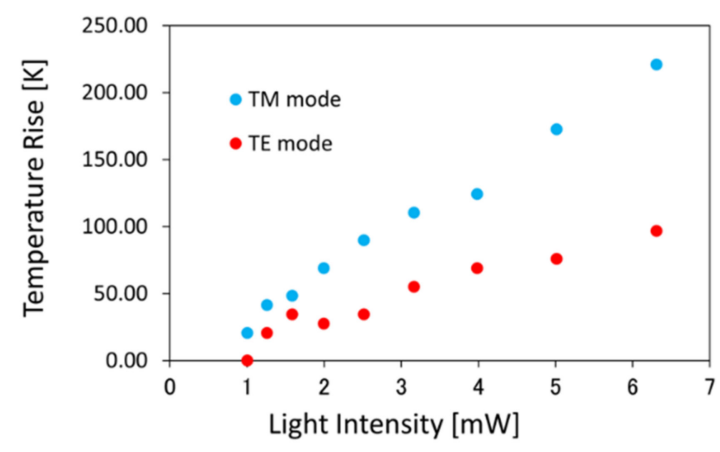

(a)

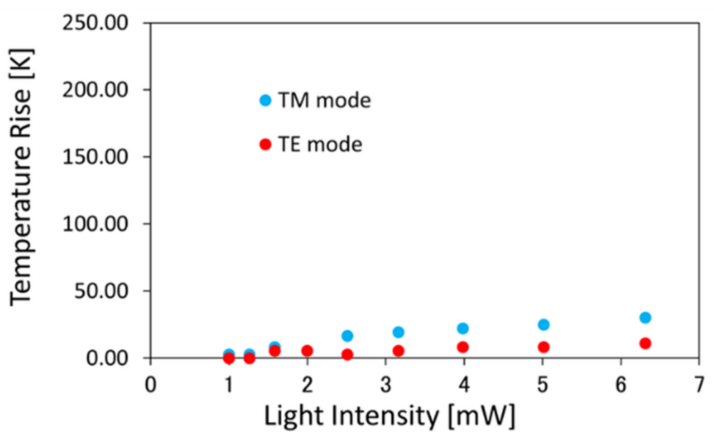

(b)

Figure 6. Estimated local temperature rise for TM and TE mode light with the Si waveguides having the waveguide width $w=(\mathbf{a}) 400 \mathrm{~nm}$, and (b) $1000 \mathrm{~nm}$.

In order to measure the rise time as well as temperature rise, we measured the transition of the resistance after light injection. We fabricated another Si plasmonic waveguide heater loaded with a Co thin film. The waveguide width is $400 \mathrm{~nm}$, and thickness of the $\mathrm{SiO}_{2}$ buffer layer is $194 \mathrm{~nm}$, which is larger than that of the device measured in Figures 2-6. The length $l$ of the Co film on top of the Si wire waveguide is $10 \mu \mathrm{m}$. The distance between the pair of electrode pads is $50 \mu \mathrm{m}$, which is smaller than that of Figures 2-6 $(200 \mu \mathrm{m})$. The temperature dependence of the resistance of the whole electrode $\frac{\Delta R_{\mathrm{C}}}{\Delta T}$ is $2.01 \times 10^{-2} \Omega / \mathrm{K}$. We measured the transition of the resistance change by a two-probe method with a digital multimeter. Figure 7 shows the transition of the resistance change after light injection with the intensity of 0 to $8 \mathrm{dBm}(1$ to $6.3 \mathrm{~mW})$ and time interval of $0.5 \mathrm{~s}$. The maximum temperature change of $0.007 \Omega$ was detected for intensity of $6.3 \mathrm{~mW}$ and $1 \mathrm{~s}$ after light injection. The transition time (1 s) is the same for the all light intensities. Measurement with shorter time interval is necessary for more precise analysis of relationship between the rise time and light intensity, which is one of the future issues. The estimated temperature rise is $44 \mathrm{~K}$, which is $1 / 5$ of that of the device described in Figure $6 a$. 


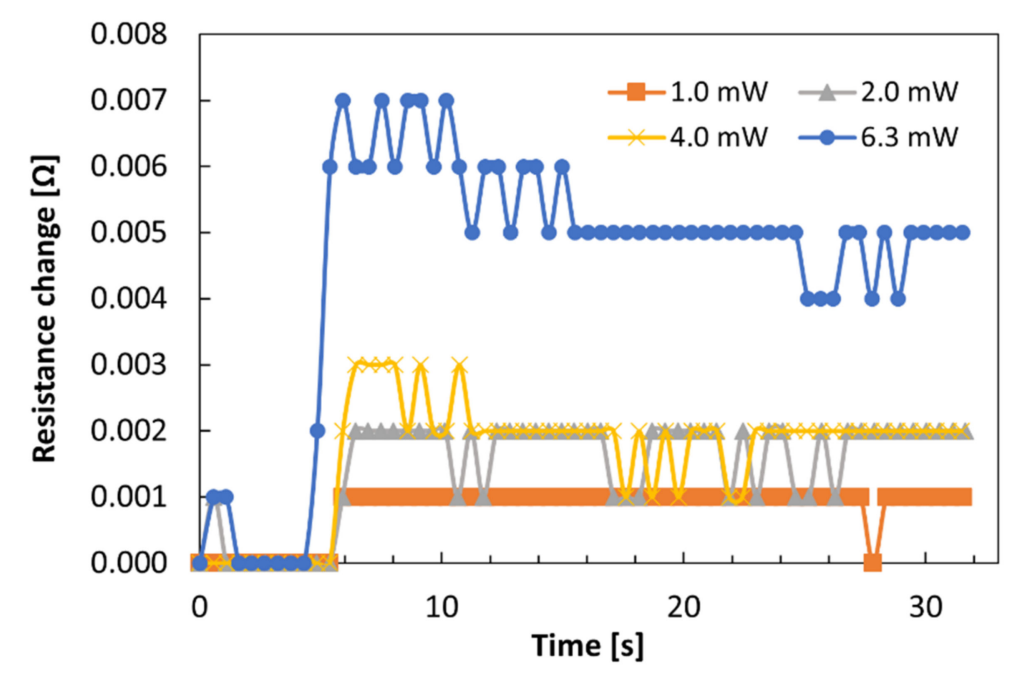

Figure 7. Transition of the measured resistance change after light injection of 1.0, 2.0, 4.0, and $6.3 \mathrm{~mW}$ for TM mode light with the $\mathrm{Si}$ waveguides with $200 \mathrm{~nm}$-thick $\mathrm{SiO}_{2}$ buffer layer. Input light was injected at $t=4 \sim 5.5 \mathrm{~s}$.

\section{Discussion}

We discuss the validity of Equation (1) for estimation of the temperature rise. The temperature dependences of the resistance of the whole electrode $\frac{\Delta R_{\mathrm{C}_{0}}}{\Delta T}$ are $7.24 \times 10^{-2} \Omega / \mathrm{K}$ for $l_{\mathrm{Co}}=200 \mu \mathrm{m}$ and $l=8 \mu \mathrm{m}$ and $2.01 \times 10^{-2} \Omega / \mathrm{K}$ for $l_{\mathrm{Co}}=50 \mu \mathrm{m}$ and $l=10 \mu \mathrm{m} . \frac{\Delta R_{\mathrm{Co}}}{\Delta T}$ is 3.6 time larger for $l_{C o}=200 \mu \mathrm{m}$ and $l=8 \mu \mathrm{m}$, compared with that for $l_{C_{o}}=50 \mu \mathrm{m}$ and $l=10 \mu \mathrm{m}$. Since $\frac{\Delta R_{\mathrm{Co}}}{\Delta T}$ is proportional to the distance between the pair of electrode pads $l_{\mathrm{Co}}$ and inversely proportional to the area of the cross section $(w \times l), \frac{\Delta R_{\mathrm{Co}}}{\Delta T}$ is five times larger for $l_{\mathrm{Co}}=200 \mu \mathrm{m}$ and $l=8 \mu \mathrm{m}$, compared with that for $l_{\mathrm{Co}}=50 \mu \mathrm{m}$ and $l=10 \mu \mathrm{m}$. The relationship of $\frac{\Delta R_{C_{0}}}{\Delta T}$ between the fabricated two samples (3.6 time) can be explained by influences of $l_{C_{0}}$ and $l$ on $\frac{\Delta R_{C_{0}}}{\Delta T}$, which provides the validity of Equation (1).

Furthermore, we discuss the influence of the polarization and waveguide width $w$ on the resistance change of the Co layer with the Si waveguides. The resistance change upon inputting the TM mode light is 2.7 times larger than that with TE mode light for $w=400 \mathrm{~nm}$. Since the propagation loss is larger for TM mode light, owing to excitation of the SPPs at the $\mathrm{Co} / \mathrm{SiO}_{2} / \mathrm{Si}$ interface with $\mathrm{TM}$ mode light, the polarization dependence of the resistance change is clear evidence of local photothermal heating at the Co-loaded Si plasmonic waveguides. $\Delta R_{\text {light }}$ at steady state upon injecting $6.3 \mathrm{~mW}$ TM mode light is $0.032 \Omega$ for $w=400 \mathrm{~nm}$, which is 2.9 times larger than that for $w=1000 \mathrm{~nm}$. The reason for larger $\Delta R_{\text {light }}$ for $w=400 \mathrm{~nm}$ is that the propagating light in the Si plasmonic waveguide is confined to the narrower region along the width direction. Figure 8 shows the profile of the horizontal component of the magnetic field (absolute value $\left|H_{y}\right|$ ) for TM-like mode light with the Si waveguides having the waveguide width (a) $w=200 \mathrm{~nm}$, (b) $400 \mathrm{~nm}$, (c) $600 \mathrm{~nm}$, and (d) $1000 \mathrm{~nm}$, calculated by the finite-difference frequency-domain (FDFD) method [23]. Figure 8e shows the definition of the mesh for calculation of the mode profile and effective refractive index. From Figure 8, the field distribution at the $\mathrm{SiO}_{2} / \mathrm{Co}$ interface becomes more uniform with decreasing the waveguide width $w$, owing to the horizontal optical confinement. The border line between uniform and non-uniform field distributions is between $w=200$ and $400 \mathrm{~nm}$. The difference of the field distribution (uniformity) brings the difference of the heating property and resistance rise. It is possible to improve the heating efficiency by reducing the width of the Si waveguide $(w=200 \mathrm{~nm})$. 


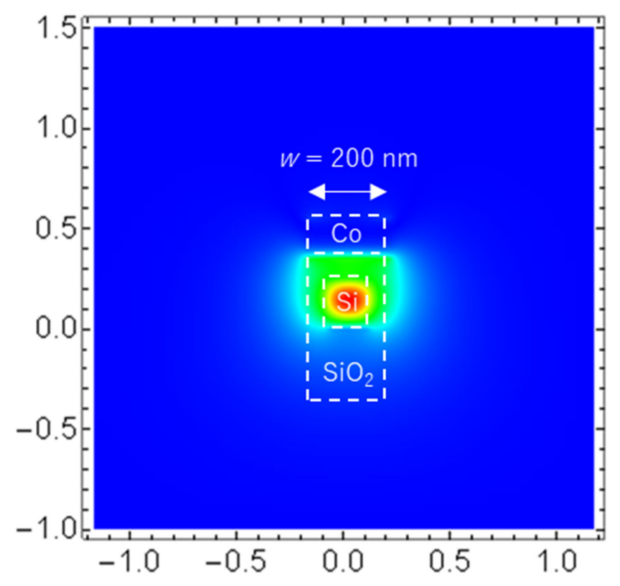

(a)

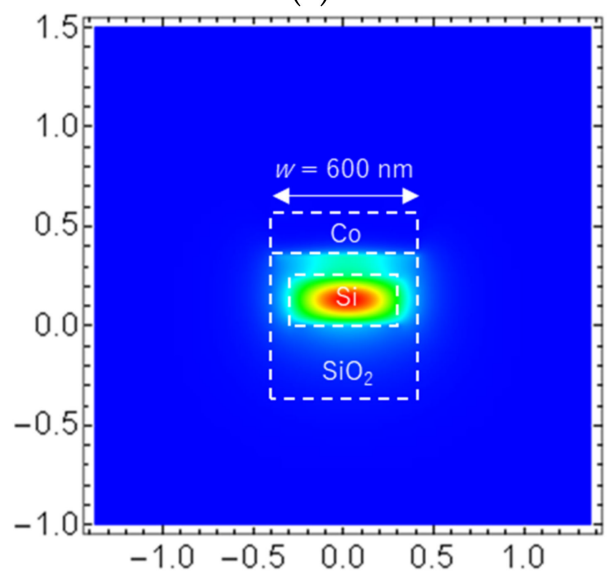

(c)

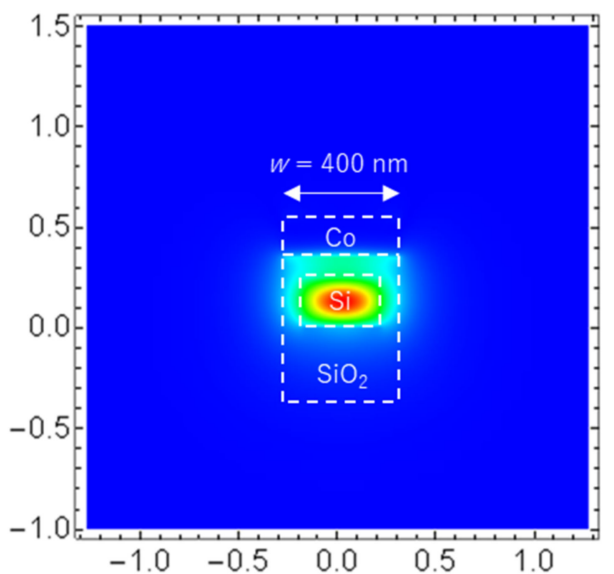

(b)

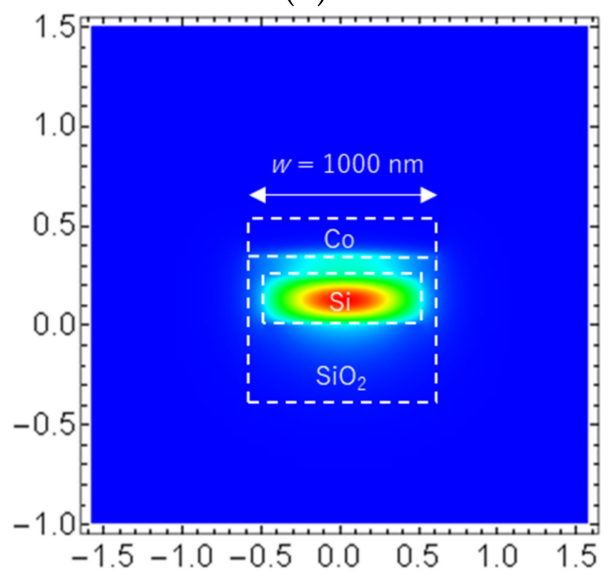

(d)

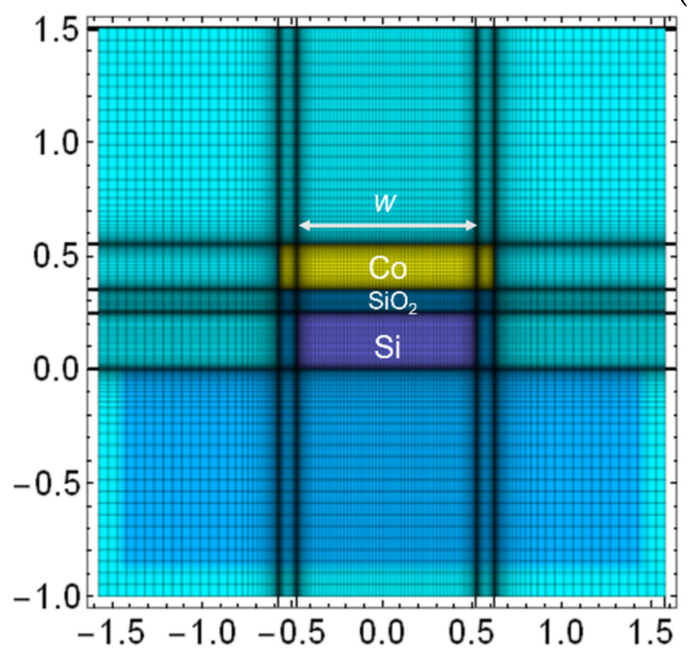

(e)

Figure 8. Calculated profile of the horizontal component of the magnetic field (absolute value $\left|H_{y}\right|$ ) for TM-like mode light with the Si waveguides for waveguide width (a) $w=200 \mathrm{~nm},(\mathbf{b}) 400 \mathrm{~nm},(\mathbf{c}) 600 \mathrm{~nm}$, and (d) $1000 \mathrm{~nm}$. (e) Definition of the mesh for calculation of the mode profile. The interfaces between the $\mathrm{Co}, \mathrm{SiO}_{2}$, and $\mathrm{Si}$ layers are shown by white dotted lines.

Estimated temperature rise of $331 \mathrm{~K}$ with $\Delta R_{\max }=0.048 \Omega$ for $w=400 \mathrm{~nm}$ was determined with sampling time interval $(1 \mathrm{~s})$ of the digital multimeter used in this study, so that smaller temperature rise is expected to be measured with a higher time resolution. Here, please note that the estimated temperature rise gives the average temperature rise of 
the Co thin film on top of the waveguide by photothermal heating, because the Co thin film is not a guided layer, and the temperature is distributed across the waveguide cross section including the $\mathrm{Co}$ thin film, $\mathrm{SiO}_{2}$ buffer layer, and $\mathrm{Si}$ core layer.

In order to reduce the required light intensity, it is important to realize uniform field distribution by narrowing the waveguide and increasing the propagation loss by decreasing the Co layer thickness, $\mathrm{SiO}_{2}$ buffer layer thickness, and $\mathrm{Si}$ core layer thickness. Influence of the Co layer thickness and $\mathrm{SiO}_{2}$ buffer layer thickness on the propagation loss for fixed Si core layer thickness of $250 \mathrm{~nm}$ and wavelength of $1550 \mathrm{~nm}$, are summarized in Table 1. The propagation loss from the Co layer increased with decreasing the thickness by exciting SPP at lower and upper interfaces of the Co layer. Thinning the Co layer is effective for decreasing the coupling loss between the Si plasmonic waveguide and Si wire waveguides, owing to smaller difference of the optical mode profiles, so that equivalent input power can be enhanced with the same input power from the input $\mathrm{Si}$ waveguide, which will contribute to a decrease of the required power level in the waveguide. Decreasing the $\mathrm{SiO}_{2}$ buffer layer thickness is effective for increasing the propagation loss. By changing the Co layer thickness from 200 to $50 \mathrm{~nm}$, and $\mathrm{SiO}_{2}$ buffer layer thickness from 100 to $10 \mathrm{~nm}$, the propagation loss can be increased to $2.9 \mathrm{~dB} / \mu \mathrm{m}$, meaning that the required light intensity is 2.9 times smaller than that of the device reported in Section 4. By decreasing the $\mathrm{Si}$ core layer thickness, it is possible to suppress the dielectric mode and enhance the field intensity at the $\mathrm{Co} / \mathrm{SiO}_{2}$ interface as hybrid mode, leading to a larger propagation loss and photothermal efficiency. The influence of the Si core layer's thickness on propagation loss is summarized in Table 2. With increasing the wavelength, size of the mode profile and field intensity at the $\mathrm{Co} / \mathrm{SiO}_{2}$ interface increase, leading to larger propagation loss and photothermal efficiency. The influence of the wavelength on propagation loss is summarized in Table 3. Narrowing the waveguide width gives smaller anisotropy and difference of the effective refractive index and mode profile between TM and TE mode light, which leads to the possibility of being less sensitive to the polarization of the input signals from the standpoint of practical optical functional device applications. By thinning the Co layer thickness, narrowing the waveguide width, decreasing the $\mathrm{SiO}_{2}$ buffer layer thickness and Si core layer thickness, enhancement of photothermal heating with shorter interaction length between the light and matter, and denser integration of Si plasmonic waveguide heaters can be expected for optical memory applications with phase change materials and HAMR.

Table 1. Calculated propagation loss in a unit of $\mathrm{dB} / \mu \mathrm{m}$ with combinations of different $\mathrm{SiO}_{2}$ buffer layer thickness $d_{\mathrm{SiO} 2}$ and Co layer thickness $d_{\mathrm{Co}}$ for fixed Si core layer thickness of $250 \mathrm{~nm}$ and wavelength of $1550 \mathrm{~nm}$.

\begin{tabular}{ccccc}
\hline \multicolumn{4}{c}{ Propagation Loss $[\mathbf{d B} / \mu \mathrm{m}]$} \\
\hline & $d_{\mathrm{Co}}=50 \mathrm{~nm}$ & $d_{\mathrm{Co}}=100 \mathrm{~nm}$ & $d_{\mathrm{Co}}=150 \mathrm{~nm}$ & $d_{\mathrm{Co}}=200 \mathrm{~nm}$ \\
\hline$d_{\mathrm{SiO} 2}=50 \mathrm{~nm}$ & 2.90 & 2.12 & 2.02 & 2.00 \\
$d_{\mathrm{SiO} 2}=100 \mathrm{~nm}$ & 1.55 & 1.08 & 1.02 & 1.00 \\
$d_{\mathrm{SiO} 2}=150 \mathrm{~nm}$ & 0.92 & 0.63 & 0.59 & 0.57 \\
$d_{\mathrm{SiO} 2}=200 \mathrm{~nm}$ & 0.55 & 0.37 & 0.35 & 0.34 \\
\hline
\end{tabular}

Table 2. Calculated propagation loss with $\mathrm{Si}$ core layer thicknesses of 200 and $250 \mathrm{~nm}$, for fixed $\mathrm{SiO}_{2}$ buffer layer thickness of $100 \mathrm{~nm}$, Co layer thickness of $200 \mathrm{~nm}$, and wavelength of $1550 \mathrm{~nm}$.

\begin{tabular}{cc}
\hline Si core Layer Thickness $[\mathrm{nm}]$ & Propagation Loss $[\mathrm{dB} / \mu \mathrm{m}]$ \\
\hline 200 & 1.67 \\
250 & 0.93 \\
\hline
\end{tabular}


Table 3. Calculated propagation loss with different wavelengths of 1350, 1450, and $1550 \mathrm{~nm}$, for fixed Si core layer thickness of $250 \mathrm{~nm}, \mathrm{SiO}_{2}$ buffer layer thickness of $100 \mathrm{~nm}$, and Co layer thickness of $200 \mathrm{~nm}$.

\begin{tabular}{cc}
\hline Optical Wavelength $[\mathrm{nm}]$ & Propagation Loss $[\mathrm{dB} / \boldsymbol{\mu m}]$ \\
\hline 1350 & 0.43 \\
1450 & 0.67 \\
1550 & 0.93 \\
\hline
\end{tabular}

\section{Conclusions}

We have reported the design, fabrication, and characterization of Co-loaded Si plasmonic waveguide heaters by measuring the resistance change. The resistance change is proportional to the input power and dependent on polarization, and inversely proportional to the waveguide width. The resistance change is measured with a transition time shorter than $1 \mathrm{~s}$. Estimated local temperature rise was $221 \mathrm{~K}$ for $6.3 \mathrm{~mW}$ TM mode light for a $400 \mathrm{~nm}$-width, $189 \mathrm{~nm}$-thick, and $8 \mu \mathrm{m}$-long waveguide. Photothermal heating efficiency was calculated as $36 \mathrm{~K} / \mathrm{mW}$. The local heating characteristics obtained in the Si plasmonic waveguides in this study can be applied for a wide range of optical device applications, including phase-change and MO materials for non-volatile optical memories, on-chip optical sensors, and a lab-on-a-chip in biology, chemistry, and medicine. Further improving of the waveguide structure measured with higher time resolution could lead to higher temperature rise, and will pave the way to a wide variety of applications from on-chip non-volatile memories to lab-on-a-chip.

Author Contributions: Conceptualization, H.S.; methodology, H.S.; software, N.O.; validation, N.O., T.M. and H.S.; formal analysis, N.O., T.M. and H.S.; investigation, N.O., T.M. and H.S.; resources, H.S.; data curation, N.O. and H.S.; writing-original draft preparation, N.O.; writing-review and editing, H.S.; visualization, N.O.; supervision, H.S.; project administration, H.S.; funding acquisition, H.S. All authors have read and agreed to the published version of the manuscript.

Funding: This work was supported by JSPS KAKENHI (Grant Number 16H04346), Japan.

Institutional Review Board Statement: Not applicable.

Informed Consent Statement: Not applicable.

Data Availability Statement: The data presented in this study are available in insert article.

Acknowledgments: The authors wish to thank Mitaro Namiki of Tokyo University of Agriculture and Technology for discussion on applications of the photothermal heaters.

Conflicts of Interest: The authors declare no conflict of interest.

\section{References}

1. Nakamura, S.; Yanagimachi, S.; Takeshita, H.; Tajima, A.; Hino, T.; Fukuchi, K. Optical Switches Based on Silicon Photonics for ROADM Application. IEEE J. Sel. Top. Quantum. Electron. 2016, 22, 3600609. [CrossRef]

2. Lira, H.L.R.; Manipatruni, S.; Lipson, M. Broadband hitless silicon electro-optic switch for on-chip optical networks. Opt. Exp. 2009, 17, 22271. [CrossRef]

3. Chen, L.; Chen, Y.K. Compact, low-loss and low-power $8 \times 8$ broadband silicon optical switch. Opt. Exp. 2012, 20, 18977-18985. [CrossRef]

4. $\quad$ Bogaerts, W.; De Heyn, P.; Van Vaerenbergh, T.; De Vos, K.; Selvaraja, S.K.; Claes, T.; Dumon, P.; Bienstman, P.; Van Thourhout, D.; Baets, R. Silicon microring resonators. Laser Photon. Rev. 2012, 6, 47-73. [CrossRef]

5. Dai, D.; Wu, H.; Zhang, W. Utilization of Field Enhancement in Plasmonic Waveguides for Subwavelength Light-Guiding, Polarization Handling, Heating, and Optical Sensing. Materials 2015, 8, 6772-6791. [CrossRef]

6. Xu, Y.; Bai, P.; Zhou, X.; Akimov, Y.; Png, C.E.; Ang, L.-K.; Knoll, W.; Wu, L. Optical refractive index sensors with plasmonic and photonic structures: Promising and inconvenient truth. Adv. Opt. Mater. 2019, 7, 1801433. [CrossRef]

7. Ye, J.; Shioi, M.; Lodewijks, K.; Lagae, L.; Kawamura, T.; Dorpe, P.V. Tuning plasmonic interaction between gold nanorings and a gold film for surface enhanced Raman scattering. Appl. Phys. Lett. 2010, 97, 163106. [CrossRef]

8. Shimizu, H.; Zayets, V. Plasmonic isolator for photonic integrated circuits. MRS Bull. 2018, 43, 425-429. [CrossRef] 
9. Richardson, H.H.; Carlson, M.T.; Tandler, P.J.; Hernandez, P.; Govorov, A.O. Experimental and Theoretical Studies of Light-to-Heat Conversion and Collective Heating Effects in Metal Nanoparticle Solutions. Nano Lett. 2009, 9, 1139-1146. [CrossRef] [PubMed]

10. Miwa, K.; Ebihara, H.; Fang, X.; Kubo, W. Photo-Thermoelectric Conversion of Plasmonic Nanohole Array. Appl. Sci. 2020, 10, 2681. [CrossRef]

11. Thamdrup, L.H.; Larsen, N.B.; Kristensen, A. Light-Induced Local Heating for Thermophoretic Manipulation of DNA in Polymer Micro- and Nanochannels. Nano Lett. 2010, 10, 826-832. [CrossRef] [PubMed]

12. Goodman, A.M.; Hogan, N.J.; Gottheim, S.; Li, C.; Clare, S.E.; Halas, N.J. Understanding Resonant Light-Triggered DNA Release from Plasmonic Nanoparticles. ACS Nano 2017, 11, 171-179. [CrossRef]

13. Paasonen, L.; Laaksonen, T.; Johans, C.; Yliperttula, M.; Kontturi, K.; Urtti, A. Gold nanoparticles enable selective light-induced contents release from liposomes. J. Control. Release 2007, 122, 86-93. [CrossRef]

14. Haffner, C.; Chelladurai, D.; Fedoryshyn, Y.; Josten, A.; Baeuerle, B.; Heni, W.; Watanabe, T.; Cui, T.; Cheng, B.; Saha, S.; et al. Low-loss plasmon-assisted electro-optic modulator. Nature 2018, 556, 483-486. [CrossRef] [PubMed]

15. Murai, T.; Shoji, Y.; Mizumoto, T. Efficient Light-to-Heat Conversion by Optical Absorption of a Metal on an Si Microring Resonator. J. Lightwave Technol. 2019, 37, 2223-2231. [CrossRef]

16. McDaniel, T.W.; Victora, R. Handbook of Magneto-Optical Data Recording: Materials, Subsystems, Techniques; Elsevier: Amsterdam, The Netherlands, 1995.

17. Zhang, H.; Zhou, L.; Rahman, B.M.A.; Wu, X.; Lu, L.; Xu, Y.; Xu, J.; Song, J.; Hu, Z.; Xu, L.; et al. Ultracompact Si-GST Hybrid Waveguides for Nonvolatile Light Wave Manipulation. IEEE Photonics J. 2018, 10, 2200110. [CrossRef]

18. Li, X.; Youngblood, N.; Ríos, C.; Cheng, Z.; Wright, C.D.; Pernice, W.H.P.; Bhaskaran, H. Fast and reliable storage using a 5 bit, nonvolatile photonic memory cell. Optica 2019, 6, 1. [CrossRef]

19. Farmakidis, N.; Youngblood, N.; Li, X.; Tan, J.; Swett, J.L.; Cheng, Z.; Wright, C.D.; Pernice, W.H.P.; Bhaskaran, H. Plasmonic nanogap enhanced phase-change devices with dual electrical-optical functionality. Sci. Adv. 2019, 5, eaaw2687. [CrossRef]

20. Kief, M.T.; Victora, R.H. Materials for heat-assisted magnetic recording. MRS Bull. 2018, 43, 87-92. [CrossRef]

21. Johnson, P.B.; Christy, R.W. Optical constants of transition metals: Ti, V, Cr, Mn, Fe, Co, Ni, and Pd. Phys. Rev. B Condens. Matter 1974, 9, 5056-5070. [CrossRef]

22. National Astronomical Observatory of Japan. Chronological Scientific Tables; National Astronomical Observatory of Japan: Maruzen, Japan, 2014. (In Japanese)

23. Kaihara, T.; Shimizu, H. Nonreciprocal dielectric-loaded plasmonic waveguides using magneto-optical effect of Fe. Opt. Express 2017, 25, 730. [CrossRef] [PubMed] 